Relations industrielles

Industrial Relations

\title{
The Canadian Labour Market par Stephen B. Peitchinis, Toronto, Oxford University Press, 1975, 367 pages.
}

\section{Bertrand Belzile}

Volume 31, numéro 4, 1976

URI : https://id.erudit.org/iderudit/028754ar

DOI : https://doi.org/10.7202/028754ar

Aller au sommaire du numéro

Éditeur(s)

Département des relations industrielles de l'Université Laval

ISSN

0034-379X (imprimé)

1703-8138 (numérique)

Découvrir la revue

Citer ce compte rendu

Belzile, B. (1976). Compte rendu de [The Canadian Labour Market par Stephen B. Peitchinis, Toronto, Oxford University Press, 1975, 367 pages.] Relations industrielles / Industrial Relations, 31(4), 685-687.

https://doi.org/10.7202/028754ar

Tous droits réservés @ Département des relations industrielles de l'Université Laval, 1976
Ce document est protégé par la loi sur le droit d'auteur. L'utilisation des services d'Érudit (y compris la reproduction) est assujettie à sa politique d'utilisation que vous pouvez consulter en ligne.

https://apropos.erudit.org/fr/usagers/politique-dutilisation/ 
in matters regarding occupational health. This dependency has resulted in numerous unnecessary diseases and deaths. Dr. Mancuso is one of several professionals providing a badly alternate source of occupational health data.

The book is intended to provide accurate, practical information to the average worker. Indeed, much of it is in the form of concise responses to letters written by concerned workers. Many first appeared in the 1974-1975 editions of the union's journal, The Machinist. There are four main sections in the book. The opening section is called "Recognizing an Occupational Illness». Exchanges such as the following occur:

Q. «I have been an auto mechanic for 40 years. I have worked in poorly ventilated shops all this time. I have also breathed brake dust from many thousands of brake jobs. I have bad legs, back, and no wind. I don't smoke cigarettes. I do smoke cigars. Is there any state compensation?

A. In brake lining dust you would have been exposed to Asbestos dust that lines the brakes. Asbestos produces a fibrosis of the lungs called asbestosis and asbestosis causes emphysema. Now, if your chest $\mathrm{x}$-rays show evidence of asbestosis, or some stage of asbestosis, then you would have the basis for an occupational disease compensation claim...»

In similar types of exchanges workers are constantly alerted to possible health hazards.

The next section discusses the question, Occupational Exposure: How Much Is Enough? It's purpose is to «Help you understand the significance of the air contaminant standard... to tell you what air sampling and testing is all about - how results are interpreted and evaluated, what the concentration levels mean; how air samples and tests identify the nature and extent of the health risks on the job and how they affect your compensation claim. » A reprint of O.S.H.A. air contaminant standards is also included.

A section on Control Measures follows, outlining, in detail, means of air contaminant control such as ventilation. Elimination of the contaminant source is judged the most desirable method, while individual worker protection the least. Owing to differences in U.S. - Canadian laws, the final section of Worker's Compensation is not very useful to Canadians.

Overall, Dr. Mancuso certainly succeeds in providing occupational health information to workers in a language they can understand. One disappointing feature of the book however, is its apolitical nature. For example, the author states, without further explanation, that the purpose of present legislated standards is to «Protect your health by specifying the limits of exposure to these toxic substances during the course of your employment. " Unfortunately, the process of standard setting is not simply a scientific exercise.

Many standards were and are inadequate by objective criteria. Morton Corn, head of O.S.H.A. recently noted the "concensus standards include many regulations which appear to have marginal, if any, effect on eliminating potential hazards in the work place. " The requirement that O.S.H.A. submit Inflationary Impact Statements on proposed regulations also reduces the likelihood that standards will be solely based on medical-scientific criteria.

In reality, the Unions, backed by scientific research, demand the safest possible standards. Companies, also backed by scientifif research, insist on the cheapest possible standards. The legislation finally enacted is determined as much by political forces as scientific data. In this sense, Patrick Kinnersly's book, The Hazards of Work, is more useful. Nevertheless, Dr. Mancuso's guide is extremely valuable. It should be read by Canadian workers.

Calgary, Alberta

Ray SENTES

The Canadian Labour Market par Stephen B. Peitchinis, Toronto, Oxford University Press, 1975, 367 pages.

Déjà un peu plus d'un an s'est écoulé depuis la parution de ce volume que l'auteur considère lui-même dans sa préface comme une édition révisée de Canadian Labour Economics publié en 1970 . Le professeur Peitchinis qualifie même cette révision de substantielle, ce qui explique sans doute le fait que le présent volume se présente comme un nouveau, non comme une édition révisée, et en plus avec un titre différent The Canadian Labour Market. Quoiqu'il en soit, le présent volume m'apparaît essentiellement une édition révisée de celui de 1970. En effet, deux seules différences 
existent entre les deux. D'abord, une partie du contenu du volume de 1970 a tout simplement été retranchée pour la publication de celui de 1975 , soit à peu près une centaine de pages (des 491). Le reste du contenu se retrouve à toutes fins pratiques inchangé dans le présent volume, à part quelques changements mineurs dont certains seront indiqués ci-dessous et, bien sûr, à part la deuxième différence annoncée plus haut, soit le rajeunissement des données statistiques. Les données les plus récentes semblent avoir été ajoutées allant de 1972 à 1974 , et peut-être exceptionnellement 1975.

Dans la présente recension, je m'efforcerai de souligner les principales différences entre les deux volumes d'une part, et des corrections ou additions qui n'ont malheureusement pas été faites selon moi, d'autre part.

Les deux premiers chapitres portant respectivement sur la population active canadienne et sur les immigrants dans celle-ci n'ont subi aucuns changements significatifs, à part bien entendu, ici comme ailleurs, le rajeunissemeit des dornées et le retranchement de 'uelques pages. Pour ma part, je dépl ce l'absence d'un traitement satisfaisant du concept économique d'offre de trava... Cett absence se fait particulièrement sentir au niveau de l'enseignement et a comme conséquence le choix d'un autre volume comme manuel de classe, même s'il s'agit d'un volume américain.

L'utilité d'un volume canadien apparaît particulièrement grande pour les deux chapitres suivants qui traitent de l'allocation de la population active (main-d'œuvre). Dans le chapitre 4, l'auteur a ajouté l'explication de V. Fuchs relativement au changement d'emploi dans les services et une section du chapitre 5 a été réécrite sur le transfert des fonctions occupationnelles.

En lisant la préface, on a l'impression que le contenu du chapitre suivant est nouveau. Mais il n'en est rien: le texte relativement à l'effet des syndicats sur l'offre et la demande de travail est demeuré inchangé de 1970 à 1975 , excepté qu'il apparaissait dans deux chapitres différents en 1970.

Quant au chapitre 7, sur le marché du travail, il eut été un endroit privilégié pour faire des changements et des additions, étant donné d'abord l'abondance de nouvelles publications au début des années 1970, étant donné aussi l'accent plus fort placé sur le marché du travail tel que suggéré par le nouveau titre du volume. C'eut été une excellente occasion de parler davantage, par exemple, de différentes classifications utiles des marchés du travail et de nouveaux modèles ou théories du marché du travail telles que la théorie radicale et celle du double marché (ou marché dual).

Les trois chapitres suivants traitent respectivement de la mobilité, de l'emploi et des effets du changement technologique sur l'emploi. Peu de changements peuvent y être notés. Il convient cependant de déplorer l'absence d'une discussion, ne serait-ce que superficielle, du phénomène nouveau de la stagflation, là où l'auteur traite de la relation d'arbitrage entre l'inflation et le chômage.

Les chapitres 11,12 et 13 se rapportent au chômage et à la lutte contre celui-ci. La section relativement aux économistes classiques face au chômage a été réécrite et en même temps abrégée. Par ailleurs, il m'apparaît un peu surprenant de ne pas trouver quelques corrections et additions ici, plus particulièrement sur le chômage structurel, sur les paiements de sécurité sociale et sur l'assurance-chômage, compte tenu des changements récents dans ces deux derniers domaines et des nouvelles études sur le premier sujet.

Le chapitre 14 sur la détermination des salaires a été modifié, essentiellement en remplaçant une présentation asse $z$ longue de la théorie de la productivité marginale par une discussion très brève du taux d'équilibre et des salaires en concurrence parfaite. Si l'on songe à l'utilisation du volume comme manuel de classe, le changement ne me semble pas très opportun. Quant au traitement des critères pratiques de détermination des salaires au chapitre 15 , aucun changement significatif n'y a été apporté. Il en est de même des chapitres 16 et 17 portant sur la structure de salaires, du chapitre 18 se rapportant à la relation entre les salaires, les prix et l'emploi, du chapitre 19 qui analyse la part du travail dans le revenu national et enfin du dernier chapitre sur l'égalité et l'inégalité des chances économiques et sociales. Dans tous ces chapitres, et en particulier dans le dernier, le lecteur aurait pu s'attendre à quelques changements.

Comme le lecteur a pu s'en rendre compte, la recension a porté essentiellement sur une comparaison des deux volumes ou éditions en mettant l'accent sur le contenu. Le rajeunissement des données statistiques en soi n'est pas une mince tâche. Ce que le soussigné déplore avant tout, c'est que l'auteur n'ait pas jugé bon de rendre compte davantage de la littéra- 
ture abondante sur les marchés du travail au début des années 1970. The Canadian Labour Market n'en demeure pas moins un ouvrage de référence fort utile, d'autant plus que les volumes du genre sont très peu nombreux au Canada.

Université Laval

Bertrand BELZILE

Brève description du système des relations du travail au Canada par André Beaucage, Ottawa, Travail Canada, 1976, $48 \mathrm{p}$.

The Current Trade Union Scene, Patterns and Trends by W. D. Wood, Kingston, Industrial Relations Center, 1976, 68 p.

Ces deux ouvrages ont de commun que, sans être analytiques, ils présentent l'un et l'autre d'une façon descriptive et succincte une mine d'informations extrêmement utiles aux personnes qui veulent connaître soit notre régime des relations du travail soit ce qui se passe actuellement dans le syndicalisme au Canada.

La brève description du système des relations du travail au Canada est une publication entièrement bilingue. Elle est destinée surtout à ceux qui cherchent à s'initier et à comprendre les mécanismes, les procédures et le fonctionnement de notre système. L'auteur, prenant comme cadre de référence le modèle Dunlop, décrit le contexte canadien, les acteurs et les mécanismes d'interaction. Si l'on peut le chicaner sur des points de détail ou des omissions comme celle de l'existence du Conseil du patronat du Québec, on y trouve l'essentiel de ce qu'il faut connaître.

The Current Trade Union Scene Patternsand Trends porte exclusivement sur le syndicalisme. L'auteur, directeur du Centre des relations industrielles de l'Université Queen, a su identifier et présenter les plus importants développements survenus dans le monde syndical du Canada au cours de 1975 ainsi qu'indique les tendances qui se manifestent. Il évoque aussi par voie de référence ce qui se passe aux États-Unis et qui peut avoir une influence chez nous. Enfin, une quinzaine de tableaux statistiques sur les divers aspects de la syndicalisation dans son ensemble de même qu'en divers milieux sont très éclairants. Cet ouvrage est indispensable à toute personne qui veut être au fait du syndicalisme au Canada.

Université Laval

Gérard DION
«Des travailleurs et des emplois », une étude du marché du travail au Canada, par le Conseil Économique du Canada, Ottawa, 1976, 289 pages.

Le rapport «Des Travailleurs et des Emplois" constitue un des tours d'horizon les plus complets du marché du travail canadien qui nous ait été présenté au cours des 10 ou 15 dernières années. Il s'agit là d'une mise à jour, dans un cadre coherent, des principales connaissances acquises sur le marché du travail canadien. Il s'agit aussi, d'une base d'information sur la réalité du marché du travail qui mise en relation avec les principaux programmes qui touchent directement ou indirectement au marché du travail pose de très sérieuses questions sur la nature et les modalités des interventions gouvernementales dans le domaine du travail. Soulignons encore le double objectif par rapport auquel on a situé l'exercice soit un marché du travail efficace et humain.

Parmi les réalités fondamentales dont le poids a pris une importance accrue et qui contribuent à modifier considérablement le fonctionnement du marché du travail et partant à remettre en question certains programmes ou du moins plusieurs de leurs modalités mentionnons:

- l'importance extrêmement considérable qu'a acquis le secteur tertiaire;

- la participation accrue de la femme au marché du travail ;

- les attitudes des jeunes face au marché du travail;

- le taux élevé de chômage et son impact particulièrement important au niveau des jeunes de même qu'au niveau de certaines régions;

- l'insatisfaction des travailleurs, malgré des revenus de plus en plus élevés, si on en juge par certains indices tel le roulement de la main-d'œuvre et les arrêts de travail;

- le fonctionnement imparfait du marché du travail qui fait qu'on assiste simultanément à du chômage élevé et à de nombreuses pénuries;

- le chevauchement du marché du travail et de la sécurité du revenu;

- le phénomène du revenu familial qui provient de la présence de deux sources de revenu dans au moins $50 \%$ des ménages et qui se situe, pour la moitié des familles frappées par le chômage, à un niveau plus élevé que pour les familles sans chômage.

De même faut-il signaler les défis qu'offrent aux politiques et programmes cer- 